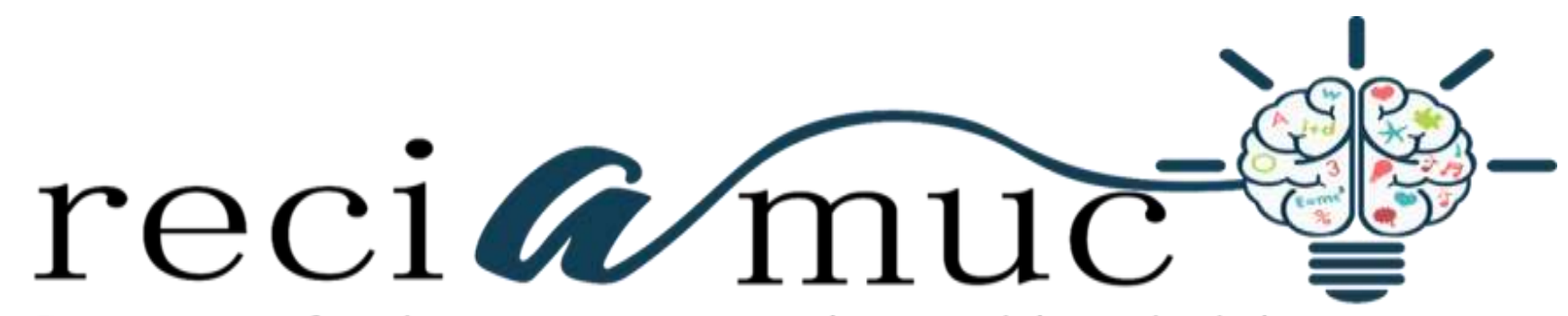

Revista científica de investigación actualización del mundo de las ciencias

Walter Manuel Mendoza Zamora ${ }^{\text {a, }}$ Tania Jaqueline Loor Mieles ${ }^{\text {b. }}$ Erika Adriana Hernández Ponce ${ }^{c}$; Shaskia Xiomara Hernández Ponce ${ }^{d}$

La calidad total como fuente de ventaja competitiva en las pequeñas y medianas empresas (Pymes) del Ecuador

Total quality as a source of competitive advantage in small and medium enterprises (SMEs) in Ecuador

Revista Científica de Investigación actualización del mundo de las Ciencias. Vol. 3 núm., 1, enero, ISSN: 2588-0748, 2018, pp. 963-984

DOI: $10.26820 /$ reciamuc/3.(1).enero.2019.963-984

URL: $\underline{\text { http://reciamuc.com/index.php/RECIAMUC/article/view/295 }}$

Código UNESCO: 5311 Organización y Dirección de Empresas

Tipo de Investigación: Artículo de Revisión

Editorial Saberes del Conocimiento

Recibido: 10/01/2019 Aceptado: 20/05/2019 Publicado: 02/07/2019

Correspondencia: walter.mendoza@unesum.edu.ec

a. Ingeniero Comercial; Magíster en Gestión Empresarial; Docente de la Universidad Estatal del Sur de Manabí; walter.mendoza@unesum.edu.ec

b. Ingeniera en Contabilidad y Auditoría; Master en Administración Pública, Mención Desarrollo Institucional; Docente de la Universidad Laica "Eloy Alfaro" de Manabí; ttanialoorm@hotmail.com

c. Ingeniera en Contabilidad y Auditoría; Magister en Tecnología Educativa y Competencias Digitales; Docente de Nivelación de la Universidad Técnica de Manabí; erikahernandez1986@ hotmail.com

d. Economista; Master Universitario en Tecnología Educativa y Competencias Digitales; Docente de Nivelación en la Universidad Técnica de Manabí; herpon_003_sx@ hotmail.com 


\section{La calidad total como fuente de ventaja competitiva en las pequeñas y medianas empresas (Pymes) del Ecuador}

Vol. 3, núm. 1., (2019)

Walter Manuel Mendoza Zamora; Tania Jaqueline Loor Mieles; Erika Adriana Hernández

Ponce, Shaskia Xiomara Hernández Ponce

\section{RESUMEN}

El presente trabajo tiene como objetivo analizar la calidad total como fuente de ventaja competitiva en las PYMES del Ecuador, teniendo en cuenta que la calidad total además de enfocarse en el producto también lo hace en el entorno organizacional, donde la pieza fundamental es el talento humano, lo que permite planificar, mantener y mejorar los procesos con eficacia y eficiencia, logrando una ventaja competitiva. Las normas ISO son establecidas por la Organización Internacional para la Estandarización estas son un conjunto de directrices para el comercio, manufactura y comunicación aplicadas a las pequeñas, medianas y grandes empresas. La calidad total en las PYMES ayuda a certificar que el producto o servicio que se ofrece al mercado cumpla con las características y normas necesarias para satisfacer las necesidades del comprador. Por otra parte, permite favorecer la sistematización de los procesos para la mejora de la organización y por ende ser competitivos en el mercado.

Palabras Claves: Calidad total, competitividad, PYMES, normas, organización. 


\section{La calidad total como fuente de ventaja competitiva en las pequeñas y medianas empresas (Pymes) del Ecuador}

Vol. 3, núm. 1., (2019)

Walter Manuel Mendoza Zamora; Tania Jaqueline Loor Mieles; Erika Adriana Hernández Ponce, Shaskia Xiomara Hernández Ponce

The present work aims to analyze the total quality as a source of competitive advantage in SMEs in Ecuador, taking into account that the total quality in addition to focusing on the product also does in the organizational environment, where the fundamental part is human talent, which allows to plan, maintain and improve the processes effectively and efficiently, achieving a competitive advantage. The ISO standards are established by the International Organization for Standardization. These are a set of guidelines for trade, manufacturing and communication applied to small, medium and large companies. Total quality in SMEs helps to certify that the product or service offered to the market meets the characteristics and standards necessary to meet the needs of the buyer. On the other hand, it allows to favor the systematization of the processes for the improvement of the organization and therefore to be competitive in the market.

Key Words: Total quality, competitiveness, SMEs, standards, organization.

\section{Introducción.}




\section{La calidad total como fuente de ventaja competitiva en las pequeñas y medianas empresas (Pymes) del Ecuador}

Vol. 3, núm. 1., (2019)

Walter Manuel Mendoza Zamora; Tania Jaqueline Loor Mieles; Erika Adriana Hernández Ponce, Shaskia Xiomara Hernández Ponce

Las pequeñas y medianas empresas (PYMES) constituyen un factor de gran relevancia para el crecimiento socioeconómico de cualquier país, lo que hace incrementar la necesidad de un desempeño y que requieran de la implantación de estrategias que beneficien al crecimiento de una empresa, para de esta manera reducir costos, mejorar la eficiencia, la calidad de los productos y por ende el incremento de la productividad.

En la gestión de calidad, los principios que intervienen, tienen gran validez para cualquier empresa, sin importar su tamaño, debido a que las PYMES con frecuencia se aproximan a los sistemas de calidad, a través, de las normas ISO 9000. Aun aceptando la validez de dichos sistemas, es de gran importancia que las empresas aprovechen todos los logros que se vayan obteniendo para poder garantizar la excelencia. Por ello, este trabajo efectúa un análisis de la implantación de la calidad total en las PYMES, demostrando de esta manera todas las conexiones existentes entre la calidad total y las normas ISO 9000.

Las ciudades del Ecuador, tienen un acelerado crecimiento y desarrollo en lo que respecta a las Pymes, pero, sin embargo, aún deben enfrentarse a una fuerte competencia que son los grandes empresarios los cuales tienen mucho poder económico. Se diferencian por su alto nivel de productividad, alcanzado por la correcta aplicación de una gestión de calidad en la administración de operaciones de su proceso, lo que ocasiona una gran desventaja en las PYMES. (Federación Nacional de Pequeñas Industrias, 2008, pág. 32)

Es evidente que las Pymes son una fuente generadora de empleo en el Ecuador, por lo que es necesario que este conjunto de pequeñas y medianas empresas logren alcanzar un desarrollo sostenible en el tiempo a través de procesos y productos de calidad que garantice mantener un campo laboral abierto y lleno de oportunidades bajo los mejores estándares de la calidad en los productos o servicios que ofrecen.

\section{Metodología.}




\section{La calidad total como fuente de ventaja competitiva en las pequeñas y medianas empresas (Pymes) del Ecuador}

Vol. 3, núm. 1., (2019) Walter Manuel Mendoza Zamora; Tania Jaqueline Loor Mieles; Erika Adriana Hernández Ponce, Shaskia Xiomara Hernández Ponce

El desarrollo de la investigación es de tipo descriptiva, documental y exploratoria. Se realizó, a través de fuentes secundarias tales como; guías bibliográficas, libros, artículos científicos, paginas oficiales de estadística de empresas en Ecuador y revistas publicados en la web. Esta información permitió analizar la calidad, el sistema de gestión de calidad y el logro de la calidad total en las Pequeñas y Medianas Empresas (PYMES) del Ecuador y como éstas se convierten en fuente de ventaja competitiva en el comercio.

Adicionalmente, la investigación es explicativa, debido a que en ella se mencionan cuál de las normas de la familia ISO 9000 es la empleada por las Pequeñas y Medianas Empresas (PYMES) en Ecuador, así como también, cuantas cuentan con la certificación y cuáles son los retos que deben enfrentar.

\section{Desarrollo.}

Calidad total como fuente de ventaja competitiva en las Pequeñas y Medianas Empresas (PYMES) del Ecuador.

El entorno más que las oportunidades suele ser el factor desencadenante para que las empresas adopten el aseguramiento de la calidad, misma que permite que las actividades, procesos productos y procesos se realicen de forma controlada y de acuerdo con las normas especificaciones y procedimientos aplicables, alcanzando altos niveles de satisfacción del cliente logrando ventajas competitivas intransferibles y duraderas a través de un sistema de gestión de calidad. (Perez, 1994)

Debido al constante cambio en la libre competencia y liberalización de las economías que vive el mundo empresarial es donde surge la calidad total misma que se proyecta como un sistema de gestión de calidad para la competitividad entre las empresas. Consecuentemente la calidad de un producto o servicio se da, a través, de la demanda la cual inicia con lo que el cliente quiere adquirir y culmina con la satisfacción del mismo, donde forman parte el proceso de producción, la organización y la participación del talento humano. 


\section{La calidad total como fuente de ventaja competitiva en las pequeñas y medianas empresas (Pymes) del Ecuador}

Vol. 3, núm. 1., (2019)

Walter Manuel Mendoza Zamora; Tania Jaqueline Loor Mieles; Erika Adriana Hernández

Ponce, Shaskia Xiomara Hernández Ponce

\section{Calidad total.}

Para Arbos (2012) "la calidad total consiste en toda la gestión de las empresas, mejorando continuamente costos, plazos, servicio y la capacidad de respuesta de la demanda, convirtiéndose en una estrategia clave para las organizaciones".

Según Manene (2010) "la calidad total son las capacidades para satisfacer las necesidades explicitas (la relación entre el cliente y proveedor) e implícitas (la manera que está posicionada en el mercado)".

La cual se enfoca no solo en el producto o servicio sino a la constante mejoría del entorno organizacional donde el talento humano juega un papel importante desde el más bajo al más alto nivel jerárquico enfocados en el logro de los objetivos empresariales y la satisfacción del cliente, por consiguiente, la implantación de un sistema de gestión de calidad permite planificar, mantener y mejorar los procesos y actividades con eficacia y eficiencia logrando ventaja competitiva. Actualmente la competitividad se basa en tres pilares fundamentales coste, tiempo y calidad llevando al cliente un producto o servicio correcto y funcional ajustándose a los requerimientos y necesidades de los consumidores a un costo y tiempo razonable.

\section{Mandamientos de la Calidad Total.}

El autor Edwards Deming (2009) afirma "Es necesario que cada individuo tenga un comportamiento adecuado conforme las actividades que este tenga que desempeñar”

Dentro de la organización existen ciertos aspectos los cuales cada miembro debe cumplir, para posteriormente obtener un producto o servicio de alta calidad diferenciándose ante la competencia y asegurando así una ventaja competitiva frente a esta, para luego ofrecerlo en el mercado.

Para Demetrio (2016) "Hoy en día para alcanzar el éxito de la organización se promueven 10 mandamientos de la calidad total", los cuales son:

1. Cumplir todas las actividades con tus clientes pase lo que pases.

2. La honestidad será nuestra base fundamental. 


\section{La calidad total como fuente de ventaja competitiva en las pequeñas y medianas empresas (Pymes) del Ecuador}

Vol. 3, núm. 1., (2019)

Walter Manuel Mendoza Zamora; Tania Jaqueline Loor Mieles; Erika Adriana Hernández Ponce, Shaskia Xiomara Hernández Ponce

3. Ser responsable con las actividades a desempeñar.

4. Reconocer los resultados obtenido en las actividades.

5. No se presentarán errores más de una vez.

6. Tratar a al talento humano como si fueran tus clientes.

7. Ser un líder que guie a los demás.

8. Compartir con el talento humano las metas alcanzadas.

9. Impulsar la responsabilidad en los trabajos en equipo.

10. Mantener las cuentas claras.

Sistema de gestión de calidad.

Según Vertice (2011) "el sistema de gestión de calidad corresponde a diversas acciones que afectan de forma positiva a toda la empresa mejorando continuamente la organización, procedimientos, procesos, recursos financieros, económicos y humanos”.

Trabajar con un sistema de gestión de calidad total conlleva a múltiples beneficios tales como; integración del trabajo en equipo, aumento de productividad, transparencia y mejora continua de los productos y servicios mediante un desarrollo de calidad de los procesos, cumplimiento de los objetivos a corto y largo plazo siguiendo las normas vigentes, además implantar un sistema de calidad total en las pymes conlleva a que la dirección o gerencia realice una planificación basándose en conocimientos filosóficos y de la empresa.

Normas ISO 9000.

Las normas ISO son las reglas para el comercio, manufactura y comunicaciones establecidas por la Organización Internacional. Estas normas son una serie de directrices aplicables a empresas grandes, medianas o pequeñas dedicada a la manufactura de cualquier producto o servicio con la finalidad de establecer un sistema de calidad mejorando los procesos. (Yanez, 2008)

La familia de las normas ISO se compone de, ISO 9000 las cuales son guía que permite la selección del tipo de modelo que se enfoque a las características que se dedique la empresa. La norma ISO 9001 se emplea cuando existe un compromiso establecido para entregar el producto 


\section{La calidad total como fuente de ventaja competitiva en las pequeñas y medianas empresas (Pymes) del Ecuador}

Vol. 3, núm. 1., (2019)

Walter Manuel Mendoza Zamora; Tania Jaqueline Loor Mieles; Erika Adriana Hernández

Ponce, Shaskia Xiomara Hernández Ponce

entre proveedores y cliente, la ISO 9002 se utiliza cuando el proveedor debe fabricar un

producto acorde a las necesidades del cliente y demostrar su correcta fabricación desde el diseño hasta la instalación, ISO 9003 se implementa cuando el producto debe pasar por diversas pruebas para que no llegue al cliente en mal estado, ISO 9004-1 se implanta cuando se desea generar confianza a la gerencia pretendiendo realizar todos los requisitos que el cliente disponga desde su el diseño hasta un servicio post-venta del producto. (Quiminet, 2006)

La competitividad impulsa a mejorar cada día debido a que los clientes al momento de adquirir un producto se inclinan por proveedores que cuenten con esta acreditación asegurándose de que la empresa que les provee cuente con un buen sistema de gestión de calidad.

Sistema de auditoría de gestión integral aplicada a las Normas ISO.

Las exigencias por mantenerse en un mercado local y global cada vez son mayores, por ende, hoy en día se ha incrementado un interés por la mejora continua de los sistemas de gestión de calidad, dentro de este ámbito las normas ISO 9000 hacen una referencia mundial para que las empresas puedan implementar estos sistemas, siendo así las auditorias, un medio para la mejora continua de los procesos permitiendo identificar los retos y oportunidades que son factores importantes para alcanzar el éxito dentro de la organización.

La auditoría se clasifica según el tipo de actividad ejecutada, pero aquí se tiene que resaltar la importancia de la auditoria dentro de los procesos, también conocida como la auditoría de controles en los procesos, que es una serie de operaciones donde se evalúan los procedimientos del trabajo que se realiza en una organización. (Parsowith, 2012, pág. 10)

Por lo tanto, la importancia de las auditorias radica, en la mejora de los procesos las cuales cada día van teniendo mayor importancia para las organizaciones pero no solo para su concepción y su puesta en práctica sino que está también debe ser implementada y evaluada dentro de la misma. Además, las auditorías externas también conocidas como auditorías de segunda, están orientadas a la evaluación que es realizada por personal técnico independiente ajeno a la organización, los cuales están encargados de la revisión de procesos actuando con sus procedimientos y tecnología. 


\section{La calidad total como fuente de ventaja competitiva en las pequeñas y medianas empresas (Pymes) del Ecuador}

Vol. 3, núm. 1., (2019) Walter Manuel Mendoza Zamora; Tania Jaqueline Loor Mieles; Erika Adriana Hernández Ponce, Shaskia Xiomara Hernández Ponce

A lo largo de los años han surgido diversos cambios en la calidad y en las normas ISO, siendo así la mejora continua en el área de calidad, en este ámbito merece destacarse que "la mejora continua es necesaria en la supervivencia económica en la economía global la cual se convierte en una mete perseguida". (Juran, J.; Godfrey, 2005, pág. 84). Está en la única vía confiable para poder sostener una ventaja en el mercado, tanto como para el cliente como para el proveedor.

Es de gran importancia señalar que todas las normas ISO mencionadas están fundamentadas en el enfoque basado en los procesos a través del desarrollo, implementación y mejora de los sistemas de gestión de una organización, medido de acuerdo a la satisfacción del cliente y el cumplimiento de sus requisitos basados en la mejora continua. (Gryna, F, 2008, pág. 122)

Pequeñas y medianas empresas (PYMES).

Antes de definir que son las Pymes se debe tomar en consideración la diferencia existente entre organización conformada por un conjunto de socios o miembros que laboran en común al logro de un mismo objetivo en relación a la empresa que son personas o individuos que laboran uniendo sus fuerzas en busca de beneficio lucrativo.

Existen diversos criterios sobre las Pymes, pero no hay una conceptualización definida de la misma, exteriorizando que el número de trabajadores es mínimo también, se las conoce como Pymes y va a depender del país donde se la establezca, su tamaño es determinado por el tiempo que lleva en el mercado, por ventas, producción, entre otros.

El autor Cleri (2007) afirma: "La pequeña y mediana empresa son por común patrimonio familiar gestionado por los mismos, por tanto, nace con la generación de idea ejecutada posteriormente por su fundador siendo pocas las empresas que inician voluntariamente".

Las Pymes son entes que ostentan diferencia a las grandes empresas y no son minúsculas ni en su etapa de inicio, solo unas cuantas llegan a expandirse y convertirse en una gran empresa el resto se mantiene en el tiempo. Se debe considerar el ciclo de vida de la empresa que va desde su nacimiento, crecimiento, madurez y muerte de la misma, es por ello que hay empresas que mantienen su razon de ser mientras que otras buscan expandirse. Sin embargo, las multinacionales o corporaciones pueden llegar a oprimir a las Pymes por lo que necesitan ser 


\section{La calidad total como fuente de ventaja competitiva en las pequeñas y medianas empresas (Pymes) del Ecuador}

Vol. 3, núm. 1., (2019)

Walter Manuel Mendoza Zamora; Tania Jaqueline Loor Mieles; Erika Adriana Hernández

Ponce, Shaskia Xiomara Hernández Ponce

apoyadas por el gobierno mediante incentivos para enfrentarse a estos feroces tigres del mercado, aunque en la mayoría son gestionadas independientemente.

\section{Calidad total en las pymes.}

"Calidad total es formar que las personas realicen de la mejor manera todas las cosas que realmente importan de cualquier carácter tienen que realizarse”. (Crosby, 2017)

Por consiguiente, Crosby (2017) manifiesta que "en las PYMES debe existir Planeación, protección y vigilancia del desempeño de los requerimientos de los compradores en la fabricación de los bienes y servicios, de esta manera lo que dice”.

El autor (Fidalgo, 2016) expresa que en la actualidad se debe crear un sistema de Gestión en las empresas por obligación, debido este tipo de sistemas está ligado a varias acciones reguladas por una o más individuos dentro de la empresa con la finalidad de administrar y vigilar los recursos.

Un sistema de Calidad ayuda a certificar que un producto o servicio pueda satisfacer las necesidades de los compradores y a su vez pueda salvaguardar sus diferencias cumpliendo con todos los requisitos necesarios, es decir, alcanzar su objetivo, por lo que la organización debe utilizar métodos importantes que deben tener soporte bien documentado, para que de esta manera todo el personal que trabaja en la misma esté capacitado y pueda elaborar las actividades con todos los procesos necesarios y obtener los resultados esperados. (GrandesPymes, 2015)

Así mismo, las empresas deben ir mejorando día a día su calidad, de esta manera lograran obtener menores precios y satisfacer las necesidades de los clientes en el mercado. Las PYMES se acercan a la eficacia por medio de los sistemas ISO 9000. De esta manera se logra que las empresas deban aprovechar todos sus beneficios que se han obtenido para que seguir con la excelencia. (Researchgate, 2011) 


\section{La calidad total como fuente de ventaja competitiva en las pequeñas y medianas empresas (Pymes) del Ecuador}

Vol. 3, núm. 1., (2019)

Walter Manuel Mendoza Zamora; Tania Jaqueline Loor Mieles; Erika Adriana Hernández Ponce, Shaskia Xiomara Hernández Ponce

Ventajas de la implantación de la calidad total en las pymes.

La Gestión De Calidad Total (GCT), es una herramienta de gestión la misma que está destinada para alcanzar altos niveles de calidad en productos o servicios dentro de una organización. "Por lo tanto, estos reciben valor añadido aumentando su satisfacción al momento de la adquisición de dicho producto o servicio" (Saez, 2007, pág. 75). Esto trata básicamente en una filosofía empresarial la cual centra su atención en la satisfacción del cliente.

La GCT es un conjunto de actividades que van conducidas hacia toda la organización para que esta pueda alcanzar de forma eficaz y eficientemente todos sus objetivos, de la misma manera que esta pueda proveer productos y servicios que satisfagan en su totalidad a los clientes en tiempo y precios establecidos. (Gonzales , 2011, pág. 80)

Actualmente, la Gestión De Calidad Total (GCT) va más allá de conseguir la satisfacción única del cliente, está más bien se empleada como sistemas de gestión de procesos para la aplicación en todas las áreas de una empresa comprendida por una estructura organizativa y hasta de la misma dirección, teniendo así un nivel de competitividad interno como un buen ambiente laboral y externo en la calidad de los productos ofrecidos.

En la gerencia moderna en lo que respectan PYMES no se puede pasar por alto un factor muy importante, que son las características que los actuales escenarios empresariales presentan, manifiestando una dinámica de competitividad en donde los requisitos fundamentales son únicamente la calidad y productividad para que estas puedan permanecer dentro de un mercado. (Mora, 2009)

Las pequeñas y grandes empresas (PYMES) no cuentan con los mismos recursos que las grandes compañías, es por ello que el recurso más importante para estas es la flexibilidad, ya que, se sienten muy vulnerables a los diferentes cambios existentes en su entorno y esto se debe a que tienen un humilde desarrollo propio.

Algunas ventajas de la implantación de la calidad en las PYMES según (Ganser, 2016) son las siguientes: 


\section{La calidad total como fuente de ventaja competitiva en las pequeñas y medianas empresas (Pymes) del Ecuador}

Vol. 3, núm. 1., (2019)

Walter Manuel Mendoza Zamora; Tania Jaqueline Loor Mieles; Erika Adriana Hernández

Ponce, Shaskia Xiomara Hernández Ponce

- Se tiene mejor contacto entre la empresa y el cliente y es más accesible conocer las necesidades del mismo.

- Permite que la toma de decisiones sea más coherente y transparente.

- La utilización de estrategias claras.

- Tener una organización de trabajo eficaz.

La competitividad de las PYMES no podría lograrse en su totalidad si estas no aplican los sistemas de calidad total. La aplicación de estos sistemas son un factor clave porque permite que cada función cuente con el personal necesario que vele por la calidad de los productos o servicios y así mejorar cada día sus procesos y procedimientos.

Las normas ISO 9000 en las Pequeñas y Medianas Empresas (PYMES).

La implementación de las normas ISO 9000 genera ventajas para las pequeñas y medianas empresas. Debido al constante cambio que vive el mercado y la globalización, las normas ISO permiten a las PYMES competir con empresas más grandes equiparándose en eficiencia, para poder cumplir con los clientes que exigen proveedores con certificación de calidad y generando confianza en ellos, gracias a las mejoras en la eficiencia y la productividad del producto o servicio, lo que brinda un valor añadido a la organización. (ISO, 2014)

Un sistema de gestión de calidad mediante las normas ISO 9000 se enfoca en las características de la propia PYME para favorecer la sistematización de los procesos en la mejora de la organización, es necesario que las mismas definan los procesos y por ende los documenten. Contar con los recursos adecuados para su correcto funcionamiento de flujo de procesos y establecer los métodos y estrategias para la mejora continua forma parte de la clave del éxito de la calidad, un punto importante es medir el desempeño del talento humano y capacitarlo.

\section{Certificación ISO 9001 para grandes medianas y pequeñas empresas.}

La norma de gestión de calidad ISO 9001 es la más utilizada en todo el mundo se estima que 176 países aproximadamente utilizan esta norma en sus empresas, esto se debe, a la relación con los sistemas de gestión de calidad y al establecimiento de procedimientos para la mejora continua, comercio de exportación y satisfacción del cliente. (EKOS, 2015) 


\section{La calidad total como fuente de ventaja competitiva en las pequeñas y medianas empresas (Pymes) del Ecuador}

Vol. 3, núm. 1., (2019) Walter Manuel Mendoza Zamora; Tania Jaqueline Loor Mieles; Erika Adriana Hernández Ponce, Shaskia Xiomara Hernández Ponce

Empresas grandes, medianas y pequeñas tanto públicas como privadas, que se dedican a diferentes actividades comerciales; tales como fabricantes y proveedores de servicios, cuentan con la certificación ISO 9001 en todo el mundo con el interés de fortalecer sus procesos y por ende ser competitivos ante la competencia.

Retos de la implantación de la norma ISO 9001 en Ecuador.

Para (SRI, 2012) indica que en el Ecuador existen empresas entre ellas pequeñas, medianas y grandes las cuales con el transcurso del tiempo se han ido expandiendo e incrementando debido a la congruencia de diversas actividades que se desarrollan en el país.

Según (E., 2016) Manifiesta que las Medianas y Pequeñas empresas en el Ecuador se dedican a la producción de bienes y servicios, generando empleo aportando al desarrollo social produciendo, comprando y demandando productos con valor añadido.

Se identifican dos factores críticos que se tienen que mantener controlados al momento de implantar un sistema de gestión de calidad; el compromiso que adquiere la alta dirección y el trabajo en equipo con los colaboradores obteniendo cada uno un 42,86\%, los beneficios obtenidos luego de la certificación es el control de procesos para las 42,86\% de las organizaciones, el cual se logra con una constante evaluación de los procesos.(EKOS, 2015)

Segmento de Pequeñas y Medianas Empresas que cuentan con certificación ISO.

Según (Servicio de Acreditación Ecuatoriano, 2017) "Los certificados ISO emitidos en el Ecuador son las más divulgadas en el país, habiendo un incremento en los últimos años”. Lo que indica que las negocios y pymes se están preocupando por mejorar la calidad de sus productos.

En un mundo tan competitivo, es necesario utilizar estrategias que permitan crecimiento de los negocios. Un ejemplo de ellos es insertar las normas ISO, tal como lo hacen ciertas pymes en el Ecuador. Ese es el caso de AMAGUA C.E.M ubicada en Guayaquil, la cual es una empresa que se dedica al servicio de agua potable y alcantarillada en el cantón Samborondón, al igual que la empresa GRAIMAN que es una empresa cuencana que elabora y comercializa pisos de cerámica, certificada desde 2001 con las normas ISO 9001. 


\section{La calidad total como fuente de ventaja competitiva en las pequeñas y medianas empresas (Pymes) del Ecuador}

Vol. 3, núm. 1., (2019)

Walter Manuel Mendoza Zamora; Tania Jaqueline Loor Mieles; Erika Adriana Hernández

Ponce, Shaskia Xiomara Hernández Ponce

Niveles estadísticos de la Pequeña y Mediana Empresa en el Ecuador.

El INEC Instituto Nacional de Estadísticas y Censo en Ecuador a través de su DIEE Directorio de Empresas y Estadísticas indica que se registraron para el año 2017 un total de 884.236 empresas lo que representó un aumento respecto al año anterior donde se establecieron cifras de 870.842. Estas empresas fueron divididas de acuerdo a su actividad económica y las cuales se encuentran registradas en el SRI, IESS y RISE; por consiguiente, se vio un aumento de 1,5\% contrario al año anterior donde se vio una notable reducción de empresas al 0,83\% (Regalado, Moya, \& Solano, 2018). Tanto como de un año a otro se ha visto una evolución significativa en el registro de nuevas empresas activas dentro del ecuador constituyendo un gran aporte a la economía del país y su desarrollo trayendo consigo plaza de trabajo disminuyendo así el desempleo.

A continuación, se exteriorizará datos obtenidas del INEC de su directorio de empresas y establecimientos:

Figuras 1. Evolución del número de empresas, período 2012-2017.

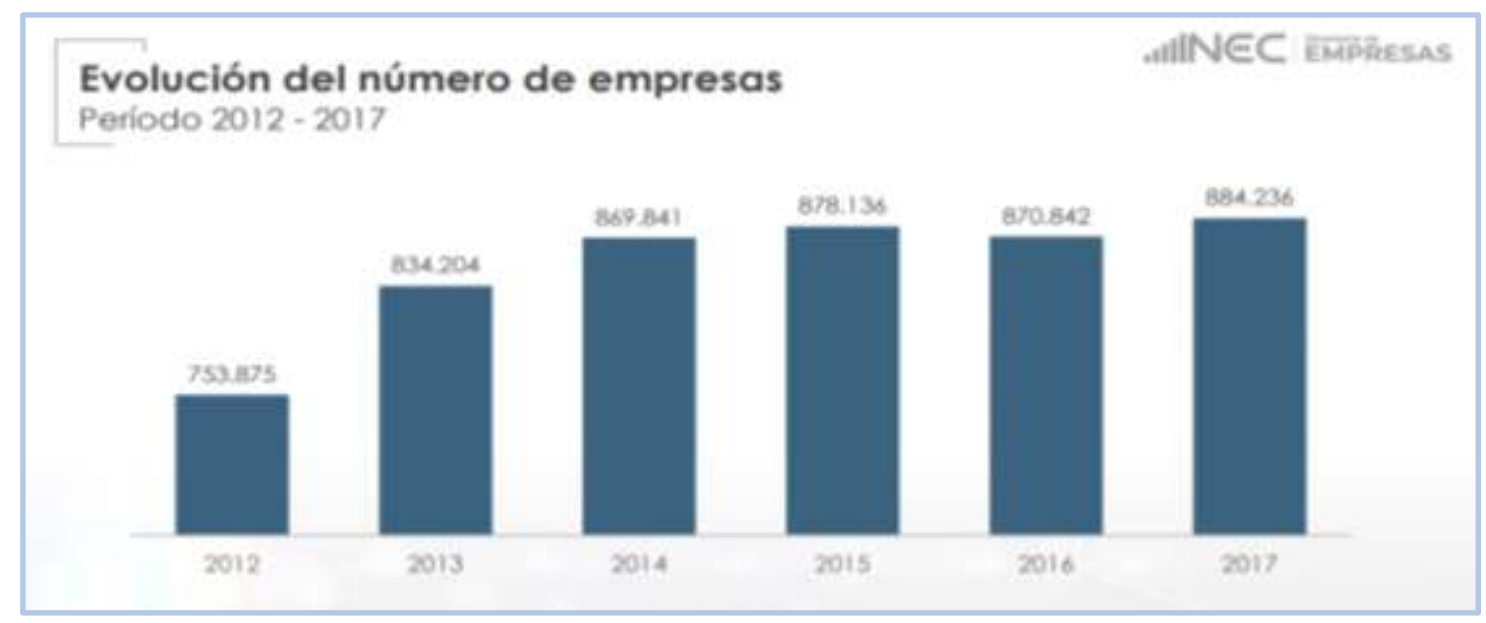

Fuente: (DIEE, 2018).

Conforme a la figura 1 se puede observar la evolución y la variación que se ha llevado a cabo desde el 2012 hasta el 2017, por lo tanto, coexistió una leve baja para el año 2016 con un registro de 870.842 empresas lo que indica que salieron del mercado ecuatoriano por diversos factores 


\section{La calidad total como fuente de ventaja competitiva en las pequeñas y medianas empresas (Pymes) del Ecuador}

Vol. 3, núm. 1., (2019)

Walter Manuel Mendoza Zamora; Tania Jaqueline Loor Mieles; Erika Adriana Hernández

Ponce, Shaskia Xiomara Hernández Ponce

que forjaron el cese o cierre de sus actividades generando bajas en la economía del país y sus habitantes aumentando en número de desempleos.

Finalmente se indica que el crecimiento de las empresas en Ecuador ha ido evolucionando en el tiempo con una variación para el año 2016 donde se observa una disminución de la misma pudiendo haberse desarrollado por factores políticos, económicos, entre otros., lo que sí que tanto como la micro, pequeña, mediana y gran empresa han sido un punto favorable dentro de la economía del país y de su producto interno bruto.

Figuras 2. Estructura de empresas según su tamaño.

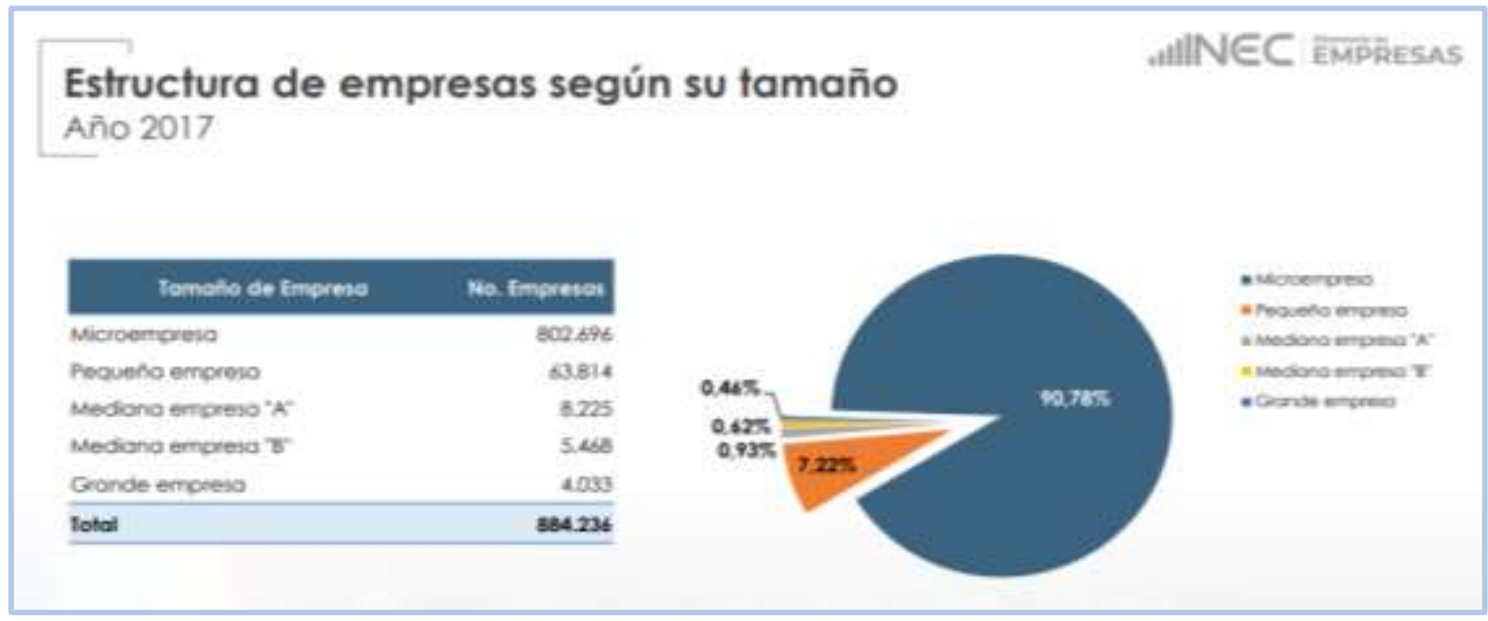

Fuente: (DIEE, 2018).

Las empresas ecuatorianas según su tamaño muestran para el año 2017 que la microempresa tuvo mayor participación en el país con un 90,78\%. Por lo tanto, el número de las grandes empresas fue de $0,46 \%$ contando con un gran número de afiliados al IEES y proporción de ventas. (Regalado, Moya, \& Solano, 2018, p. 6)

En consecuencia, existe una gran importancia de las microempresas dentro de Ecuador en relación a la pequeña empresa que solo aportan un 7,22\% lo que indica que no tiene una gran participación en el mercado local frente a las microempresas donde la producción es baja, debido al carácter familiar, uní personal o de agrupación, formando parte de la Ley de economía popular y solidaria. Con estos datos estadísticos se puede indicar, que el gobierno debería prestar más atención a este sector económico brindándole más oportunidades de financiamiento u otro 


\section{La calidad total como fuente de ventaja competitiva en las pequeñas y medianas empresas (Pymes) del Ecuador}

Vol. 3, núm. 1., (2019)

Walter Manuel Mendoza Zamora; Tania Jaqueline Loor Mieles; Erika Adriana Hernández

Ponce, Shaskia Xiomara Hernández Ponce

beneficio con la finalidad de elevar el desarrollo del país. En los actuales momentos no existen aún datos estadísticos actualizados para determinar si en el año 2018 hubo un incremento o disminución de las empresas en ecuador.

Figuras 3. Estructura de empresas por sector económico. Período 2012-2017.

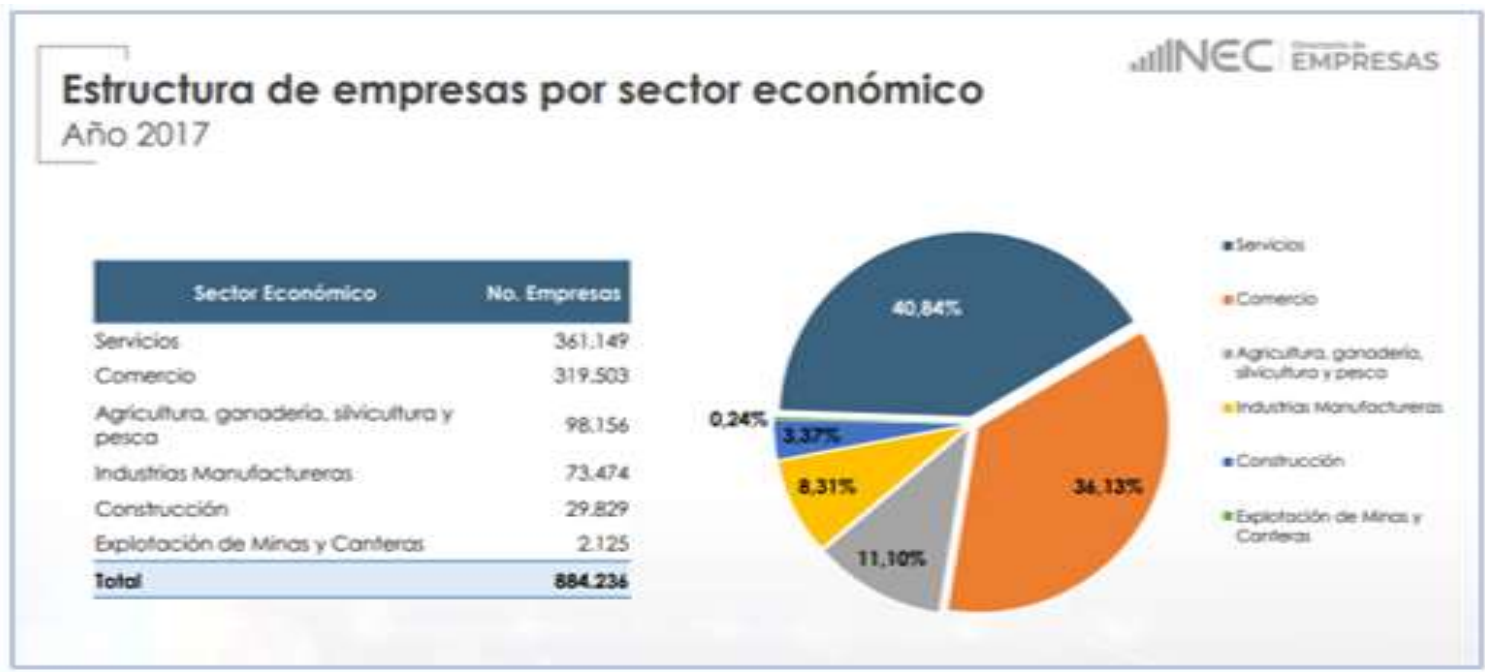

Fuente: (DIEE, 2018).

Figuras 4. Estructura de empresas por actividad económica.

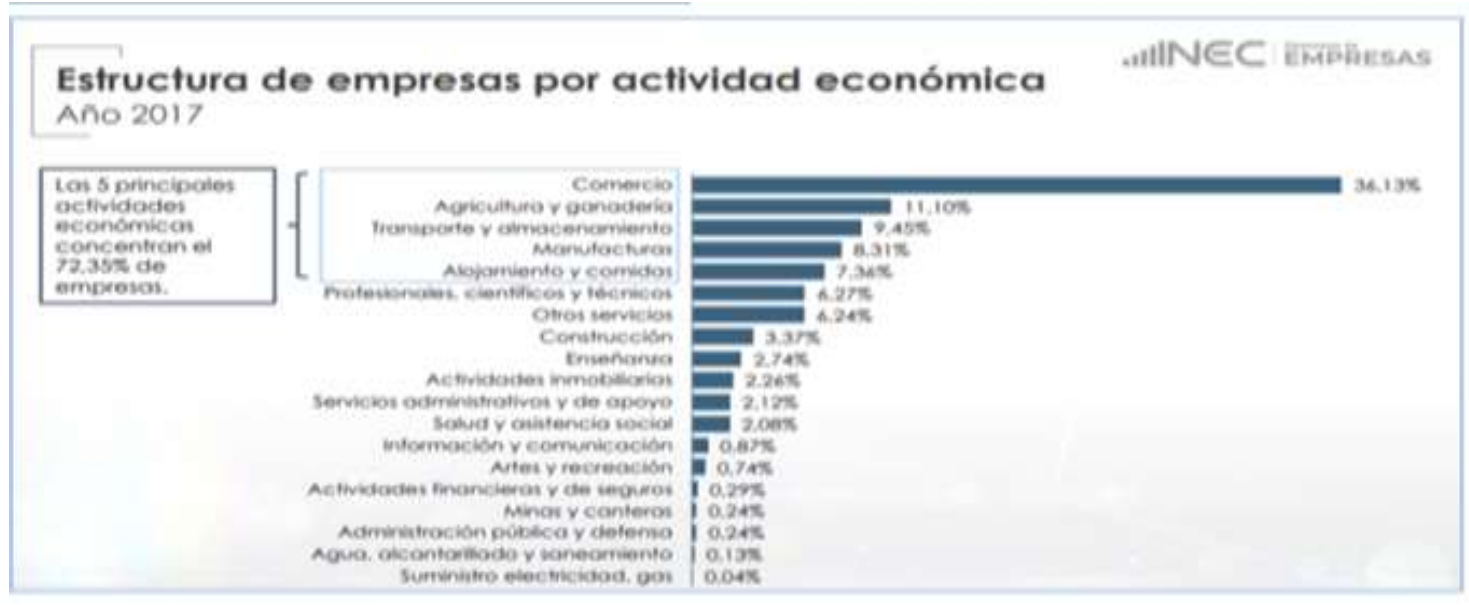

Fuente: (DIEE, 2018).

En cuanto a la estructura del DIEE por sector o actividad económica muestra que los servicios cuentan con un 40,84\% mientras que el comercio con un $36,13 \%$ siendo los sectores que mayor 


\section{La calidad total como fuente de ventaja competitiva en las pequeñas y medianas empresas (Pymes) del Ecuador}

Vol. 3, núm. 1., (2019)

Walter Manuel Mendoza Zamora; Tania Jaqueline Loor Mieles; Erika Adriana Hernández

Ponce, Shaskia Xiomara Hernández Ponce

actividad concentraron en el año 2017. El sector con menor concentración fue la explotación de minas y canteras con apenas 0,24\%. (Regalado, Moya, \& Solano, 2018, p. 6)

Hay que hacer énfasis, que el comercio juega un papel importante para el sector económico registrándose 319.503 empresas a nivel nacional no estando tan debajo de las empresas del sector servicio con 361.149, centrándose el crecimiento económico en estas áreas y aportando al PIB del país; los restantes sectores, aunque no sean fuente primordial se le debería dar más cabida a estas áreas donde se podría explorar más sus recursos y se generaría mayor inversión y producción para el país.

En conclusión, es de suma importancia para el país la realización de actividades económicas como la de servicios y de comercio que generen tanto por entidades particulares como públicos escenarios de desarrollo a la nación. Por tal razón, el gobierno debería brindar una economía más abierta con menos restricciones a las empresas, que ofrezca escenarios de seguridad para los inversionistas para poder aumentar la producción de innovación aunado a un excelente sistema de calidad.

Figuras 5. Estructura de empresas según provincia.

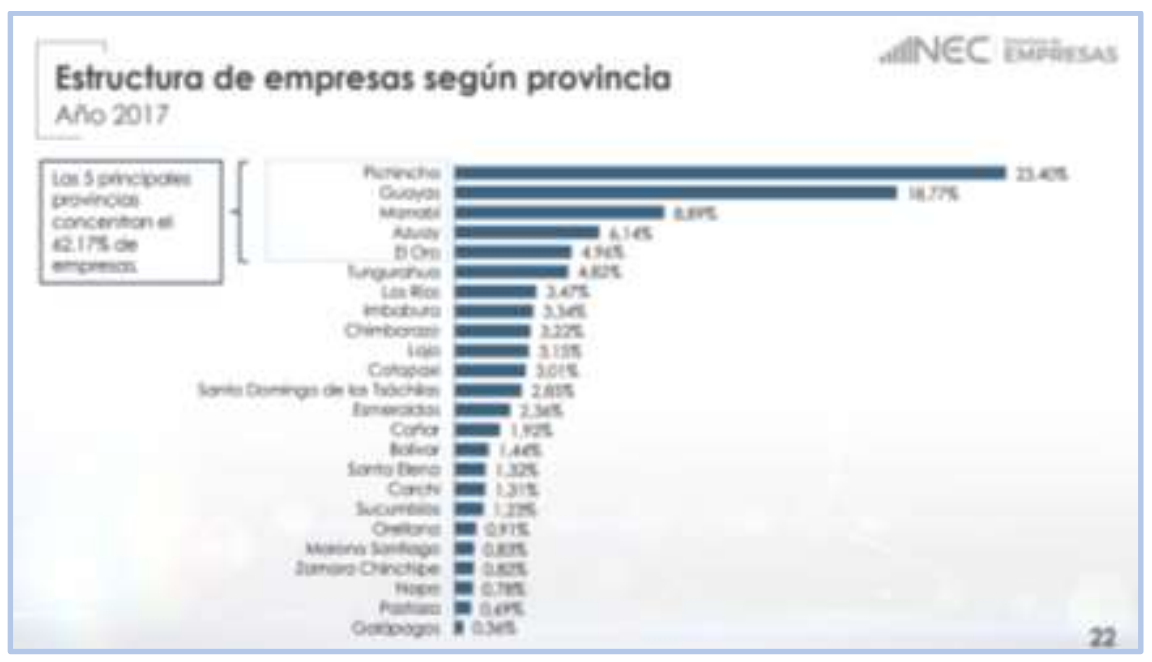

Fuente: (DIEE, 2018). 


\section{La calidad total como fuente de ventaja competitiva en las pequeñas y medianas empresas (Pymes) del Ecuador}

Vol. 3, núm. 1., (2019)

Walter Manuel Mendoza Zamora; Tania Jaqueline Loor Mieles; Erika Adriana Hernández

Ponce, Shaskia Xiomara Hernández Ponce

Como se observa en la figura 5 la estructura de las empresas según su provincia la que mayor número de empresas registran se estable en un top tres de las principales ciudades que cuentan con mayor demanda de las mismas estando Pichincha con un $23.40 \%$, seguida de guayas con un $18.77 \%$ y Manabí $8.33 \%$, por lo tanto existe un margen significativo de empresas establecidas en la sierra que en la costa a lo que podríamos indicar que el nivel de trabajo se desarrolla en la sierra con mayor desarrollo generando inversión.

Finalmente se indica que el mayor número de creación y registro de empresas se da en las provincias de Pichincha, Guayas y Manabí por ser ciudades con mayor capacidad de espacio y alto número de población bastante, así mismo, pocas de ellas afilian a sus trabajadores o les brindan los beneficios de ley, dando lugar un nivel bastante significativo de trabajadores informales y desempleo.

Figuras 6. Participación en ventas según tamaño de empresa.

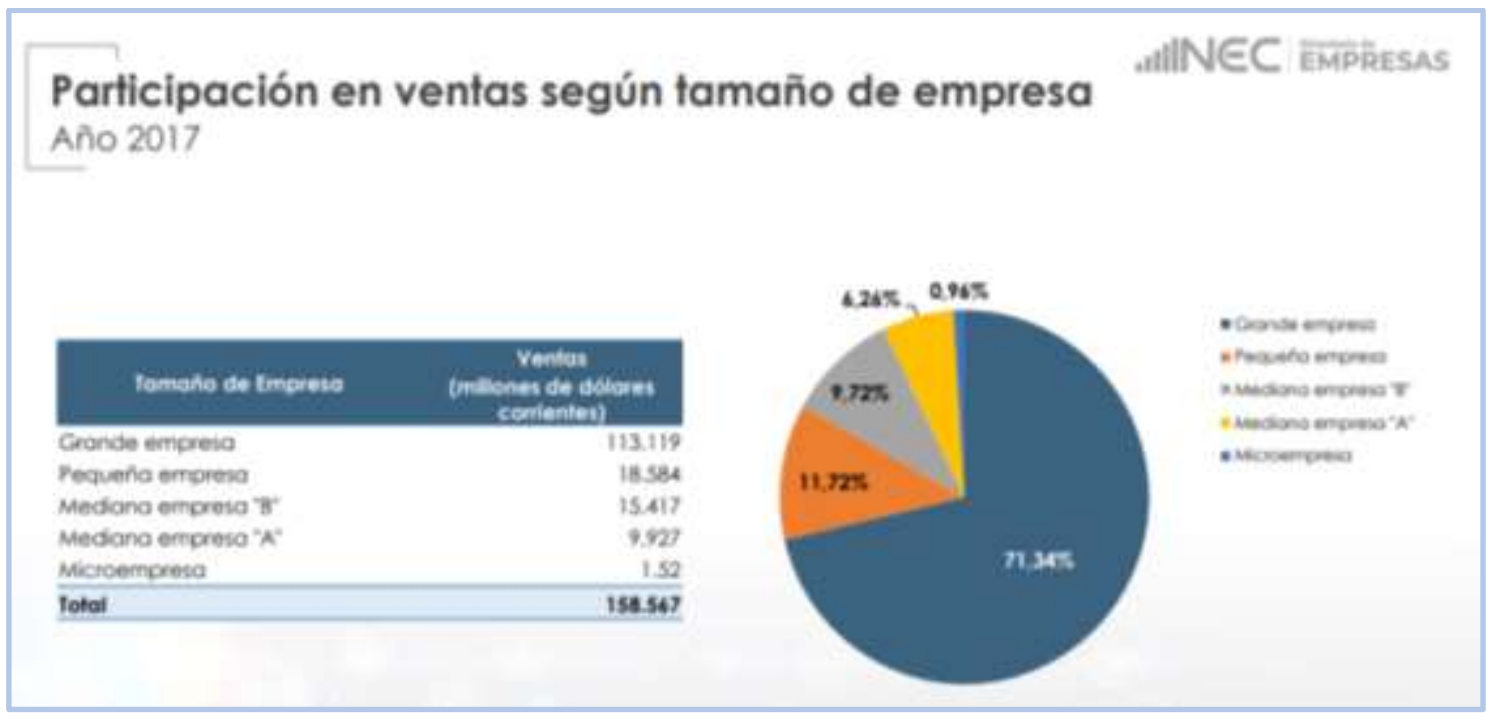

Fuente: (DIEE, 2018).

Por consiguiente, la participación en ventas según su tamaño muestra que la grande empresa tiene participación en el mercado centrándose con un 71,34\% mientras que la mediana empresa A y B cuenta con un 15,98\% en el 2017. Y en relación a la figura 2 que se muestra el nivel de registro de empresas en donde las microempresas cuentan con 802.696 a diferencia de las grandes empresas con 4.033, por lo que a veces no importa el número de empresas registradas si 


\section{La calidad total como fuente de ventaja competitiva en las pequeñas y medianas empresas (Pymes) del Ecuador}

Vol. 3, núm. 1., (2019) Walter Manuel Mendoza Zamora; Tania Jaqueline Loor Mieles; Erika Adriana Hernández Ponce, Shaskia Xiomara Hernández Ponce

no se ve un incrementado en sus niveles de ventas como es el caso de las microempresas que solo apenas generaron 1,52 millones de dólares con apenas 0,96\% en el 2017, siendo las grandes empresas las que aprovechan el comercio concibiendo ventas de 113.119 millones de dólares sin contar con muchas empresas registradas en el Ecuador.

En conclusión, el nivel de ventas no depende del que tantas empresas se registren en el país, como se puedo observar en los gráficos que la gran empresa a pesar de no contar con muchas representa un porcentaje contundente en participación comercial dentro del país todo esto podría cambiar si el gobierno apoyara más a las micro, pequeña y mediana empresas a generar más ventas aprovechando que existe un gran número de ellas.

La calidad total en las Pymes Ecuatorianas les permite el mejoramiento continuo, analizando y comprendiendo las necesidades, posibles expectativas y satisfaciendo los requerimientos de los clientes tanto externos e internos. Garantizando a largo plazo el crecimiento, la supervivencia y la rentabilidad de la empresa la cual se logra a través, de la participación de todos los colaboradores con liderazgo transformando los servicios, productos, procesos y cultura para logro de las metas.

\section{Conclusiones.}

De acuerdo a la revisión bibliográfica realizada se observó en el desarrollo del trabajo investigativo, estadísticas que llevaron al análisis de la calidad total de las pequeñas y medianas empresas del Ecuador y de la importancia en la aplicación de las Normas ISO 9000 para la a generación de estrategias competitivas que dan lugar al desarrollo de las empresas y al avance de la nación.

La calidad Total no solo se basa en la satisfacción de las necesidades y expectativas que tiene el cliente con los productos o servicios que se ofrecen, sino también en el mejoramiento de la organización donde el talento humano debe enfocarse en cumplir todos los objetivos empresariales y así mismo poder satisfacer a sus clientes. 


\section{La calidad total como fuente de ventaja competitiva en las pequeñas y medianas empresas (Pymes) del Ecuador}

Vol. 3, núm. 1., (2019)

Walter Manuel Mendoza Zamora; Tania Jaqueline Loor Mieles; Erika Adriana Hernández Ponce, Shaskia Xiomara Hernández Ponce

En el desarrollo de la investigación se mostró, un significativo aumento de empresas registradas en el país bajo las normas ISO para el año 2017 a nivel nacional, mayormente se establecieron en las principales ciudades de Pichincha, Guayas y Manabí donde su población es bastante alta, con micro empresa que cuentan con un porcentaje del $90.78 \%$.

Las Pequeñas y Medianas Empresas en el Ecuador constituyen un aporte fundamental para el desarrollo del país, brindando fuentes de trabajo y contribuyendo de esta manera la disminución del desempleo, siendo una ventaja competitiva la aplicación del sistema de gestión de calidad.

\section{Bibliografía.}

Camisón y González . (2011). Implantacion de calidad. Gestion y Gerencia , 77.

Arbos, L. C. (2012). Gestion de la Calidad Total. Madrid, España: Diaz de Santos. Recuperado el 09 de 02 de 2019, de http://books.google.com.ec

Cleri, C. (2007). PYMES estado circunstacncial de un ciclo de vida y particularidades. En C. Cleri, El libro de las PYMES (págs. 37- 41). Argentina: Ediciones Granica S.A.

Crosby, P. (12 de Agosto de 2017). HerramientasPYMES. Obtenido de HerramientasPYMES: $\mathrm{http}: / /$ herramientaspyme.com/calidad-total/

Demetrio., S. P. (2016). Gestiopolis. Obtenido de https://www.gestiopolis.com/los-10mandamientos-de-la-calidad-de-gestion/

DIEE. (Octubre de 2018). Directorio de empresas y establecimientos 2017. Obtenido de INEC Directorio de empresas : http://www.ecuadorencifras.gob.ec/documentos/webinec/Estadisticas_Economicas/DirectorioEmpresas/Directorio_Empresas_2017/Documen tos_DIEE_2017/Documentos_DIEE_2017/Principales_Resultados_DIEE_2017.pdf

E., X. Z. (16 de 06 de 2016). Eumet.net. Recuperado el 15 de 02 de 2019, de Las PYMES en el Ecuador: www.eumed.net 


\section{La calidad total como fuente de ventaja competitiva en las pequeñas y medianas empresas (Pymes) del Ecuador}

Vol. 3, núm. 1., (2019)

Walter Manuel Mendoza Zamora; Tania Jaqueline Loor Mieles; Erika Adriana Hernández Ponce, Shaskia Xiomara Hernández Ponce

\begin{tabular}{llllllllll}
\hline EKOS. $\quad(07$ & de 2015). & Recuperado el 16 & de & 02 & de & 2019, & de
\end{tabular} http://www.ekosnegocios.com/revista/pdfTemas/1259.pdf

Federación Nacional de Pequeñas Industrias. (2008). las PYMES afiliadas a la año 2008 - I FASE - PYMES COSTA Y ORIENTE”. LA IMPORTANCIA DE LAS PYMES EN EL ECUADOR , 32.

Fidalgo, C. A. (2016). Docplayer. Recuperado el 27 de 02 de 2019, de https://docplayer.es/10774168-.html

Ganser. (2016). Calidad total. Sistema de Gestión de la Calidad. 120.

Gonzales . (2011). El Deming Prize Committee de la Union of Japanese Scientists and Engineers. 80 .

GrandesPymes. (3 de Agosto de 2015). Obtenido de GrandesPymes: https://www.grandespymes.com.ar/2015/08/03/calidad-total/

Gryna, F. (2008). Análisis y planeación de la calidad. Quinta Edición. Mexico : Mc Graw Hill.

ISO. (05 de 12 de 2014). blog de calidad ISO. Recuperado el 16 de 02 de 2019, de Normas ISO en las PYMES: http://blogdecalidadiso.es/normas-iso-en-las-pymes/

Juran, J.; Godfrey. (2005). Manual de Calidad de Juran. Volumen I. McGraw Hill. Madrid. España. Directrices para la auditoría de gestión de la calidad y/o ambiental.

Manene, L. (01 de 12 de 2010). Luis Manene. Recuperado el 09 de 02 de 2019, de Calidad Total: www.luismiguelmanene.com

Mora. (2009). PYME con respecto a la calidad y la productividad. Obtenido de http://www.degerencia. com/articulo.

Ochoa, S. $\quad$ (s.f.). $\quad$ ekosnegocios. Obtenido de http://www.ekosnegocios.com/revista/pdfTemas/1259.pdf 


\section{La calidad total como fuente de ventaja competitiva en las pequeñas y medianas empresas (Pymes) del Ecuador}

Vol. 3, núm. 1., (2019)

Walter Manuel Mendoza Zamora; Tania Jaqueline Loor Mieles; Erika Adriana Hernández

Ponce, Shaskia Xiomara Hernández Ponce

Parsowith. (2012). Auditorías, Mejora Continua y Normas ISO: factores clave para la//. 10.

Perez, J. (1994). Gestion de la calidad empresarial. Madrid: ESIC editorial. Recuperado el 15 de 02 de 2019, de https://books.google.com.ec

Quiminet. (05 de 01 de 2006). Recuperado el 05 de 02 de 2019, de ¿Que son las normas ISO 9000?: htt://www.quiminet.com

Regalado, A., Moya, L., \& Solano, B. (31 de Octubre de 2018). Estadísticas de las empresas. Obtenido de Directorio de empresas y establecimientos DIEE: http://www.ecuadorencifras.gob.ec/documentos/web-

inec/Estadisticas_Economicas/DirectorioEmpresas/Directorio_Empresas_2017/Documen tos_DIEE_2017/Documentos_DIEE_2017/Boletin_Tecnico_DIEE_2017.pdf

Researchgate. (15 de Febrero de 2011). Obtenido de Researchgate: https://www.researchgate.net/publication/28050877/la_gestion_de_calidad_total_en_la_P YME_a_partir_de_los_sitemas_ISO_9000/

Saez. (2007). Modelosparalaimplantacióndelagestióndecalidadtota. Gestion y gerencia, 75.

Servicio de Acreditación Ecuatoriano. (Septiembre de 2017). Obtenido de http:/www.acreditacion.gob.ec/encuesta-iso-2016/

SRI. (20 de 11 de 2012). Recuperado el 15 de 02 de 2019, de http://.sri.gob.ec

Vertice, E. (Ed.). (2011). La gestion economica y laboral en las PYMES. Vertice. Recuperado el 09 de 02 de 2019, de books://google.com.ec

W. Edwards Deming. (2009). "Out of the Crisis". Obtenido de http://genesismex.org/ACTIDOCE/CURSOS/CHILE-CO-OT'10/LIBROS/ROBERTOFUERA\%20DE\%20LA\%20CRISIS.pdf

Yanez, C. M. (05 de 12 de 2008). Gestion de calidad en base a la norma Iso 9001. Internacional eventos, 2. Recuperado el 30 de 01 de 2019, de www:internacionaleventos.com 